Chemical Society, The Minerals, Metals \& Materials Society, The American Ceramic Society, and American Society for Engineering Education. He serves as an editorial board member of the Journal of Physics: Condensed Matter and NPJ Computational Materials. Rondinelli is also chair of the Argonne Center for Nanoscale Materials Users' Executive Committee (2016-2019).

Hong Wang is currently a chair professor and vice dean of the College of Engineering at the Southern University of Science and Technology, China. Previously, she was a professor at Xi'an Jiaotong University. She received her

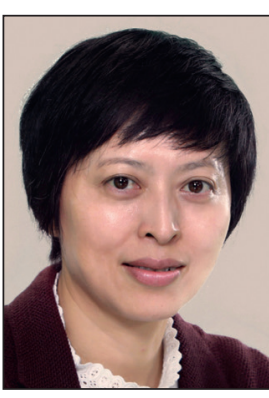

$\mathrm{PhD}$ degree in electronics materials and devices from Xi'an Jiaotong University. Wang's main research interests include dielectric materials, multifunctional composites, and dielectric measurements. She has received many honors, including the Distinguished Young Scholars Award from the National Natural Science Foundation of China (2010), Chang Jiang Scholars Award from the Ministry of
Education of China (2011), and the Scientific Innovation Leaders of the National 10000 Talents Program (2016).

Wang has (co)-authored more than 240 papers, 28 patents, and has delivered more than 50 invited talks at international conferences. She is the chair of the Asian Electroceramic Association (AECA). She is a member of the IEEE Ferroelectrics Committee. She also serves as an associate editor for IEEE Transactions on Ultrasonics, Ferroelectrics, and Frequency Control, and is an editorial board member for the Journal of Advanced Ceramics, the Journal of the Chinese Ceramic Society, and IET Nanodielectrics.

\title{
14th International Symposium on Macrocyclic and Supramolecular Chemistry
} will be held in Italy https://ismsc2019.eu

The 14th International Symposium

1 on Macrocyclic and Supramolecular Chemistry (ISMSC2019) will be held in Lecce, Italy, on June 2-6, 2019.

ISMSC2019 will provide a forum to discuss all aspects of macrocyclic and supramolecular chemistry and also topics on materials and nanoscience. This symposium will be a unique opportunity for researchers to brainstorm and promote cross-disciplinary collaborations that will have an impact on their research and related fields of applications (e.g., biomedical engineering, food science, energy storage, biosensing). It will also offer networking opportunities among peers, recognized leaders in the field, young scientists, and students. The program will include lectures from the 2016 Nobel laureates in Chemistry, from the 2019 IzattChristensen Award, Cram-Lehn-Pedersen Prize, and Sessler Early Career Researcher
Prize winners. ISMSC2019 will also host the Natural Product Reports Emerging Investigator Lectureship. Lectures, flash presentations, and poster presentations will also be selected from submissions. Scholarships covering registration fees are available for select young scientists.

Abstract submissions are due January 15 , and early bird registration ends February 15. Visit https://ismsc2019.eu for more details.

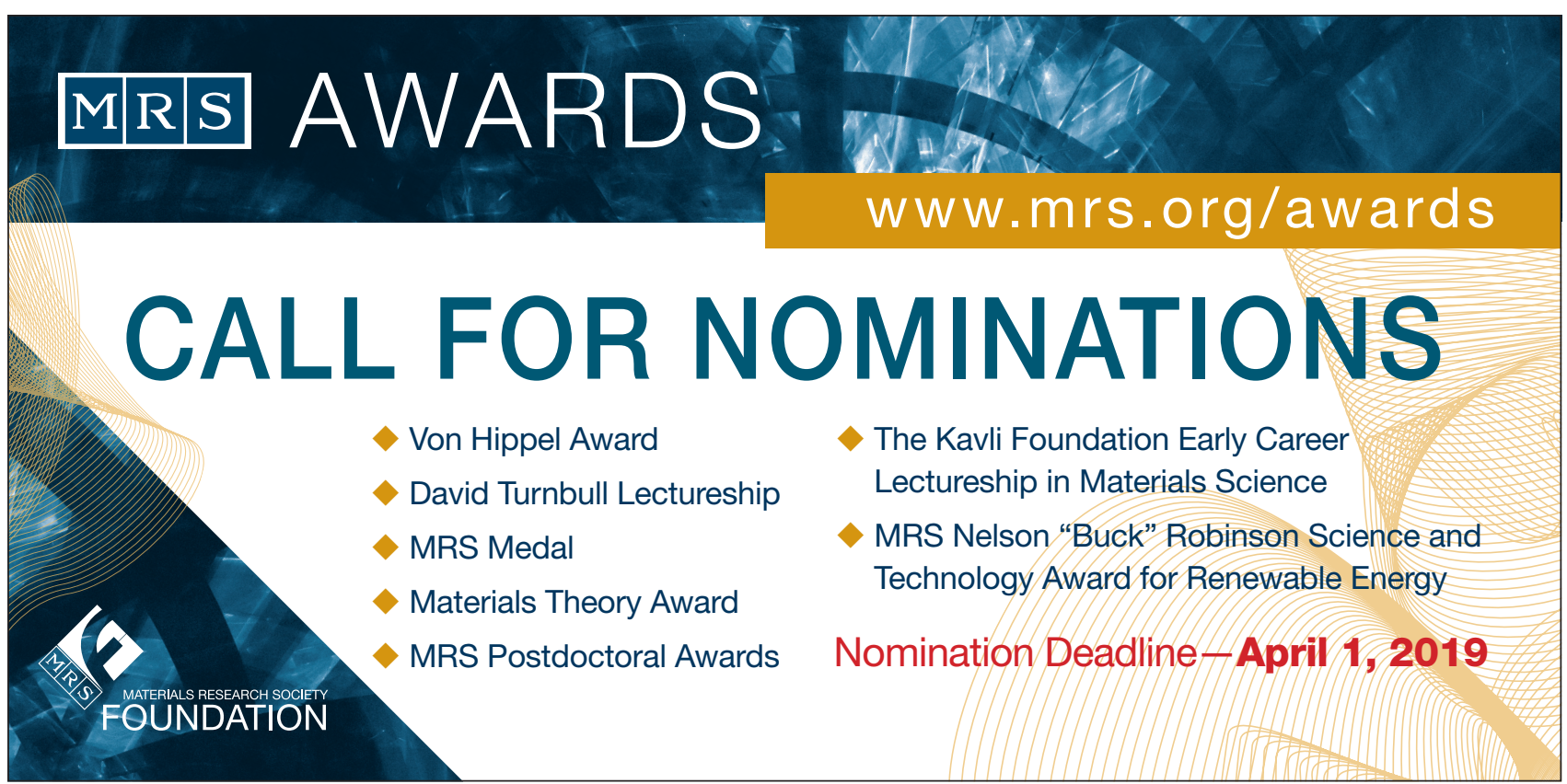

